\title{
A small-specimen investigation of the fracture toughness of $\mathrm{Ti}_{5} \mathrm{Si}_{3}$
}

\author{
KYU SUNG MIN*, A. J. ARDELL, S. J. ECK, F. C. CHEN \\ Department of Materials Science and Engineering, University of California, Los Angeles, \\ CA 90095, USA
}

\begin{abstract}
The fracture toughness of the refractory hardmetal $\mathrm{Ti}_{5} \mathrm{Si}_{3}$, with a grain size between 5 and $6 \mu \mathrm{m}$, was measured using the controlled-flaw method in conjunction with the miniaturized disc-bend test. The specimens used in these experiments were $3 \mathrm{~mm}$ diameter and varied in thickness from 150-450 $\mu \mathrm{m}$. They were indented using a Vickers pyramid indentor to indention loads varying from 2.9-79.2 N. Indentation cracking was experienced at all indentation loads, and $R$-curve behaviour was exhibited. The fracture toughness was determined to be $2.69 \pm 0.21 \mathrm{MPa} \mathrm{m}^{1 / 2}$ using a straightforward graphical procedure involving an empirical $R$-curve equation. This value is almost $30 \%$ higher than that of similar material $\left(2.1 \mathrm{MPa} \mathrm{m}^{1 / 2}\right)$ with a larger grain size, suggesting that the fracture toughness of this material, which fractures intergranularly, might be grain-size dependent.
\end{abstract}

\section{Introduction}

Traditional techniques for measuring the fracture toughness of ceramic materials, such as the single-edge notched beam, the double-cantilever beam and double torsion tests [1], are reliable, but require relatively large specimens. In a research and development programme the quantity of material available is often quite limited, due to synthesis on a laboratory scale and possible difficulties in processing.

One method of measuring the mode I fracture toughness, $K_{\text {Ic }}$, of brittle materials that has gained popularity in the past decade or so, is the controlledflaw, or indentation-flaw method, the modern theoretical foundation which was established by Chantikul et al. [2]. This method involves the introduction of a crack via Vickers microhardness indentation under a known indentation load, $F$, and measuring the fracture stress, $\sigma_{f}$, typically in four-point bending. Data are then acquired on the dependence of $\sigma_{\mathrm{f}}$ on $F$ over as large a range of $F$ as possible. The fracture mechanics of this test has been developed so that reliable values of $K_{\mathrm{Ic}}$ can be obtained. Also, if the material exhibits rising $R$-curve behaviour, i.e. if the resistance to crack extension increases with increasing crack length, analysis of the data permits evaluation of the crack-extension resistance at "infinite" crack length, $K_{\infty}$, which is generally taken as equivalent to $K_{\text {Ic }}$. The controlled-flaw method, therefore, has an advantage over the related so-called "indentation-toughness" method [3] in its ability to investigate rising $R$-curve behaviour. Additionally, it is possible to estimate $K_{\infty}$ without measuring crack lengths.
The typical specimen dimensions in the kind of controlled-flaw test discussed above are the order of tens of millimetres. These are still very large if only gram-sized quantities of material are available, and it is therefore of considerable value to develop a reliable method of measuring $K_{\infty}$ that utilizes even smaller quantities of material. We have developed just such a method over the past few years, namely a miniaturized disc-bend test (MDBT) [4,5], and have used it successfully to measure the fracture toughness of several ceramics $[6,7]$, as well as the brittle ordered intermetallic compound $\mathrm{Ni}_{3} \mathrm{Ge}$ [8]. The specimens used in these investigations are discs $3 \mathrm{~mm}$ in diameter and range in thickness from $250-400 \mu \mathrm{m}$. They are thus considerably smaller than any of the specimens used in the traditional fracture toughness testing of ceramics.

The requirements of high-performance aerospace materials at high operating temperatures, along with low density and improved oxidation resistance, have led to the consideration of refractory metal silicides as candidate materials. The microstructure and mechanical properties of one such silicide, $\mathrm{Ti}_{5} \mathrm{Si}_{3}$, were recently investigated by Rosenkranz et al. [9], who reported a room-temperature $K_{\text {Ic }}$ of approximately 2.1 $\mathrm{MPam}^{1 / 2}$. That work, therefore, provides the foundation for another comparison between the capabilities of the MDBT and more conventional methods of fracture toughness testing, and on a somewhat different class of material, because $\mathrm{Ti}_{5} \mathrm{Si}_{3}$ is intermediate in behaviour between ordered intermetallic compounds and compounds such as carbides, nitrides, etc., as noted by Rosenkranz et al. In this paper we report

* Present address: W. M. Keck Laboratory of Engineering Materials, California Institute of Technology, Pasadena, CA 91109, USA.

₹ Present address: Department of Materials Science and Engineering, University of Michigan, Ann Arbor, MI 48109-2136, USA. 
the results of our experiments using the MDBT to measure $K_{\infty}$ of $\mathrm{Ti}_{5} \mathrm{Si}_{3}$.

\section{Experimental procedure}

The material investigated in the analysis was a slab of HIPed polycrystalline $\mathrm{Ti}_{5} \mathrm{Si}_{3}$, approximately $5 \mathrm{~mm} \times$ $30 \mathrm{~mm} \times 50 \mathrm{~mm}$ in size, with a grain size between 5 and $6 \mu \mathrm{m}$, obtained from the Max Planck Institut für Eisenforschung, Dusseldorf, Germany. The Vickers hardness was measured using a Tukon microhardness tester. From twelve measurements at six different indentation loads, the Vickers hardness was determined to be $1189 \pm 86 \mathrm{~kg} \mathrm{~mm}^{-2}(11.65 \pm 0.84 \mathrm{GPa})$. This is somewhat higher than the value reported by Rosenkranz et al. [9] $\left(968 \pm 30 \mathrm{~kg} \mathrm{~mm}^{-2}\right)$. The difference is most likely related to the difference in grain size between the material we tested and that of the $\mathrm{Ti}_{5} \mathrm{Si}_{3}$ intermetallic compound tested by Rosenkranz et al. (20-50 $\mu \mathrm{m}$ cf. 5-6 $\mu \mathrm{m}$ ). Because Vickers indentation involves local yielding due to the contact stress, our alloy should be harder, considering also that the diagonal of the smallest indentation in our work (at $F=2.94 \mathrm{~N}$ ) was about $26 \mu \mathrm{m}$. The typical indentations were therefore much larger than the grain size in our alloy.

In preparing the disc specimens, slices $0.5-1 \mathrm{~mm}$ thick were cut from the slab, depending on the final desired thickness, using an electric-spark discharge machine. Discs $3 \mathrm{~mm}$ in diameter were cut from the slices with an abrasive slurry cutter. The dises were then ground and polished with $0.05 \mu \mathrm{m} \gamma$-alumina powder using standard metallographic polishing procedures. Each sample was examined to ensure that there were no pre-existing flaws. The final specimens were between 350 and $450 \pm 1 \mu \mathrm{m}$ thick, as determined by measurement with a precision digital micrometer.

Vickers indentations were placed in the centres of the discs using the aforementioned Tukon microhardness tester. The centres were located to within $\pm 10 \mu \mathrm{m}$ prior to indentation using the optical micrometer stage built into the hardness tester. All samples exhibited indentation cracks.

At least four specimens were tested at each indentation load. After testing to failure, each sample was examined to see whether the Vickers indentation served as the origin of failure. In successful tests the specimen usually broke into four pieces, with the cracks originating at the corners of the indentation. In unsuccessful tests the specimen broke into a random number of pieces; this happened more frequently at smaller indentation loads, and the data from such tests were not included in subsequent analysis.

The discs were placed in the lower die of the MDBT fixture $[4,5]$, with the indented side subject to biaxial tensile loading, and tested in the ring-on-ring mode [5]. The load was applied using a table model Instron testing machine with a crosshead speed of $0.83 \mu \mathrm{m} \mathrm{s}^{-1}$. The applied load, $P$, was measured by a $45.4 \mathrm{~kg}$ load cell and the displacement was measured with a linear variable differential transformer (LVDT). The signals from the LVDT and the load cell were received by an IBM $\mathrm{PC}$ with a data acquisition rate of 100 data points per second.

\section{Results}

3.1. Measurement of the fracture stress, $\sigma_{\mathrm{f}}$ Representative curves of applied load, $P$, versus displacement of the centre of the disc-shaped specimen, $w$, are shown in Figs 1 and 2. The curves in Fig. 1 are the type normally obtained, wherein the load increases until the specimen fails catastrophically, as indicated by the point labelled X in Fig. 1a. Failure is followed by a sudden rapid displacement of the LVDT, which occurs too rapidly for the data acquisition to record. The curve of $P$ versus $w$ at small displacements is shown in Fig. 1b, and is characterized by a typical non-linear region at small loads $[4,5]$ followed by a nearly linear region until the point of fracture. The highest load before fracture, $P_{\mathrm{f}}$, was used in calculation of fracture stress.
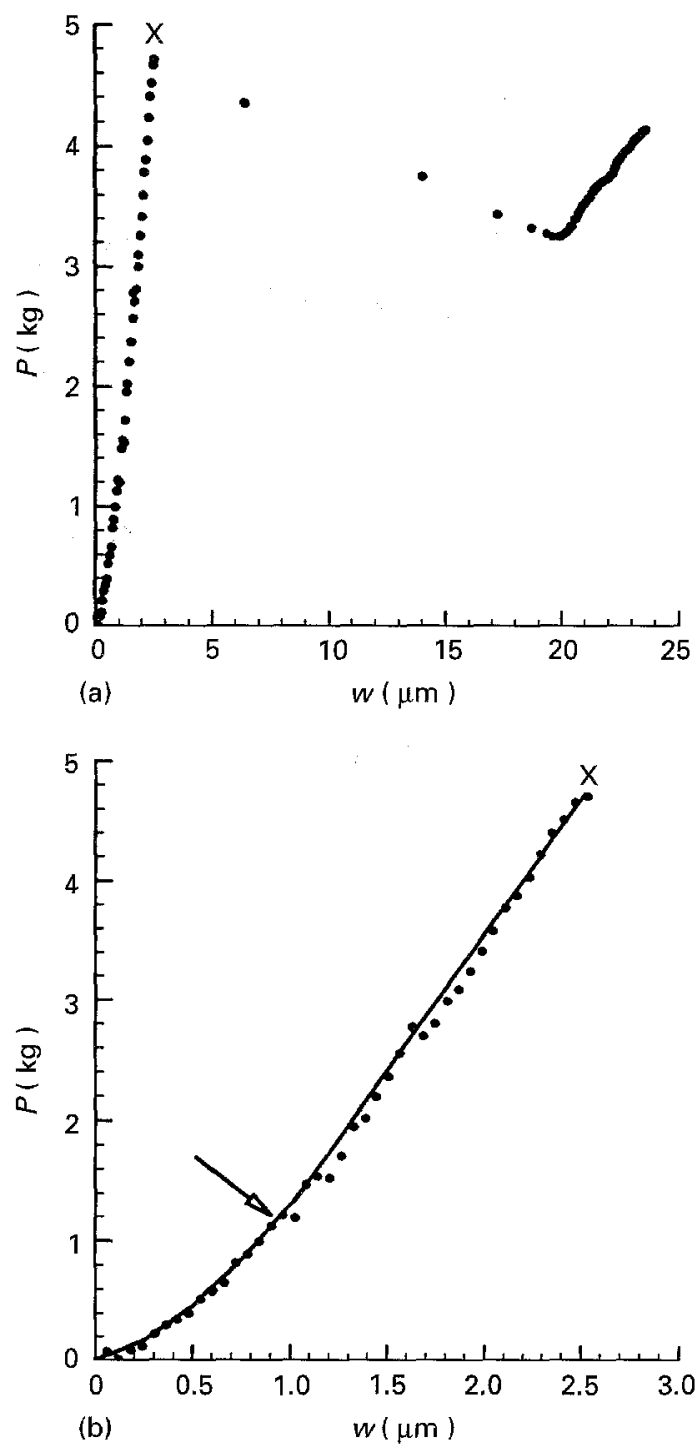

Figure 1 (a) Curve of applied load, $P$, versus displacement, $w$, for a specimen $385 \mu \mathrm{m}$ thick, indented with a load of $19.6 \mathrm{~N}$. (b) The initial portion of the curve in (a), illustrating the non-linear behaviour often observed at low applied loads. The arrow in (b) indicates the load subtracted to obtain the net load at fracture. $(x)$ The onset of fracture. 

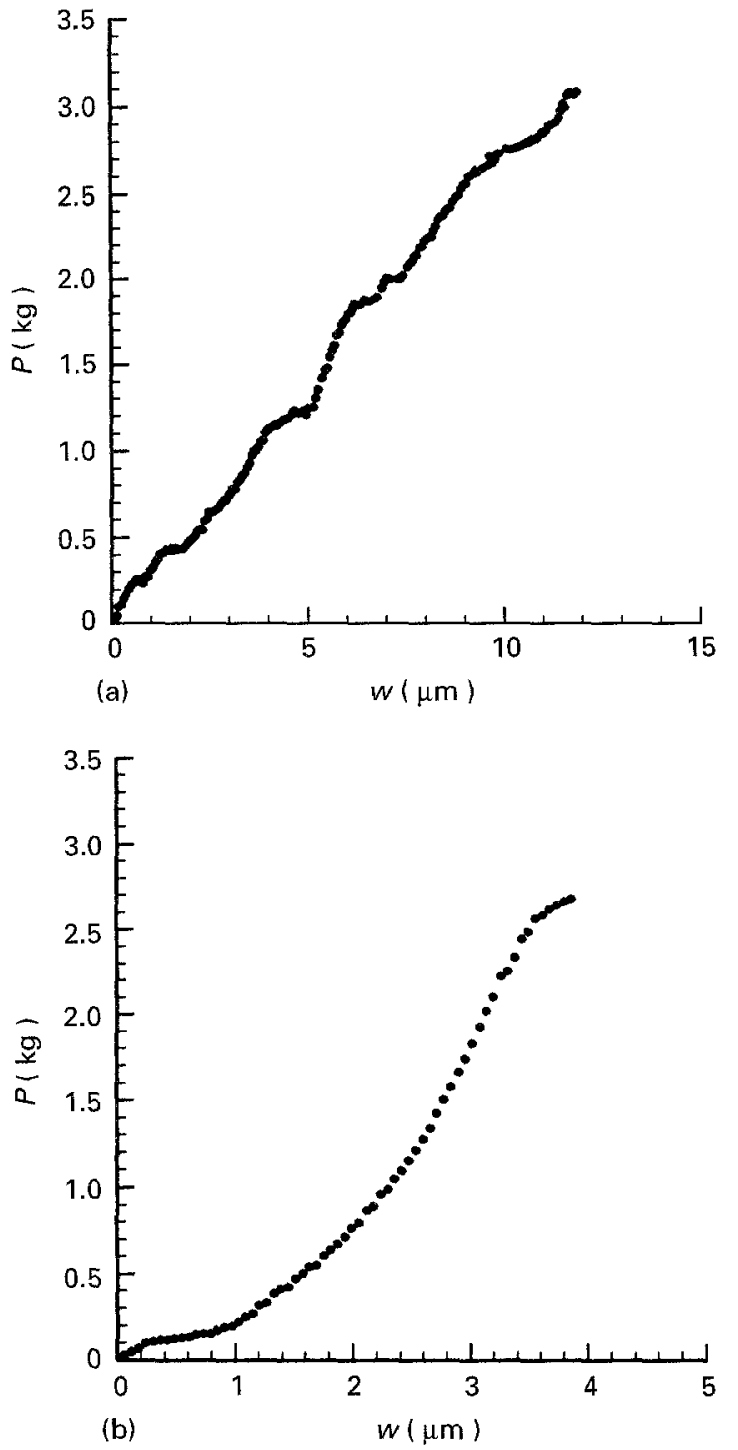

Figure 2 Examples of unusual curves of applied load versus displacement. Indentation load: (a) $F=29.4 \mathrm{~N}$, (b) $F=63.7 \mathrm{~N}$.

Not all the specimens produced curves of $P$ versus $w$ were as well behaved as that in Fig. 1. Some specimens exhibited behaviour not previously encountered, examples of which are shown in Fig. 2. The curves in Fig. 2 are not linear, and because all the specimens were tested to failure it was impractical to interrupt the tests to determine the reasons for the unusual behaviour observed. In tests such as these, $P_{\mathrm{f}}$ was taken as the maximum load recorded, and was used to calculate $\sigma_{\mathrm{f}}$ without correction.

The fracture stress was calculated from the fracture load, $P_{\mathrm{f}}$, using the formula [10]

$$
\sigma_{\mathrm{f}}=\frac{3 P_{\mathrm{f}}}{2 \pi t^{2}}\left[(1+v) \ln \frac{a}{b}+\frac{1-v}{2}\left(1-\frac{b^{2}}{a^{2}}\right) \frac{a^{2}}{R^{2}}\right]
$$

where $v$ is Poisson's ratio, $t$ is the specimen thickness and $a, b$, and $R$ are the radii of the lower die, the loading ring, and the specimen, respectively. Young's modulus, $E$, and the shear modulus, $G$, are reported [9] as 156 and $60.8 \mathrm{GPa}$, respectively, so that Poisson's ratio, $v$, is estimated to be 0.283 .

\subsection{Evaluation of the fracture toughness}

The fracture stresses of unindented specimens exhibited considerable scatter, the average values and standard deviations being $199.8 \pm 95.6 \mathrm{MPa}$ for nine specimens tested. There were no apparent reasons for this, but the scatter exhibited by the indented specimens was significantly smaller. The dependence of $\sigma_{\mathrm{f}}$ on $F$ for the indented specimens is shown in Fig. 3. The slope of the straight line drawn through the log-log plot is -0.215 , which is larger than $-1 / 3$, indicating $R$-curve behaviour for this material.

The fracture mechanics methodology behind the controlled-flaw test is well established [2], so it is reviewed only briefly here. The stress intensity factor impelling the extension of a penny-like crack of radial dimension $c$, initially produced by the indentor under load $F$, is given by [2]

$$
K_{\mathrm{e}}(c)=K_{\mathrm{a}}(c)+K_{\mathrm{r}}(c)=\psi \sigma c^{1 / 2}+\frac{\chi F}{c^{3 / 2}}
$$

where $\sigma$ is the applied (far field) stress, $\psi$ is a dimensionless constant determined by the crack geometry and $\chi$ is an elastic-plastic constraint constant given by the equation [11]

$$
\chi=\delta\left(\frac{E}{H}\right)^{1 / 2}
$$

where $E$ is Young's modulus, $H$ is the Vickers hardness, and $\delta$ is a constant. By modelling the indentation crack as a wedge-loaded half-penny crack, Shetty et al. [12] derived the following expression for $\delta$

$$
\delta=\frac{\psi}{24(1-2 v)(\sqrt{2} \pi \tan \phi)^{2 / 3}}
$$

where $2 \phi$ is the apex angle of the Vickers indentor $\left(\phi=68^{\circ}\right)$.

The constant $\psi$ depends on the dimensions of the specimen and the size and shape of the crack. The equilibrium shape of the surface crack generally changes as the crack extends from its initially semicircular shape to a semielliptical one, for which the ratio

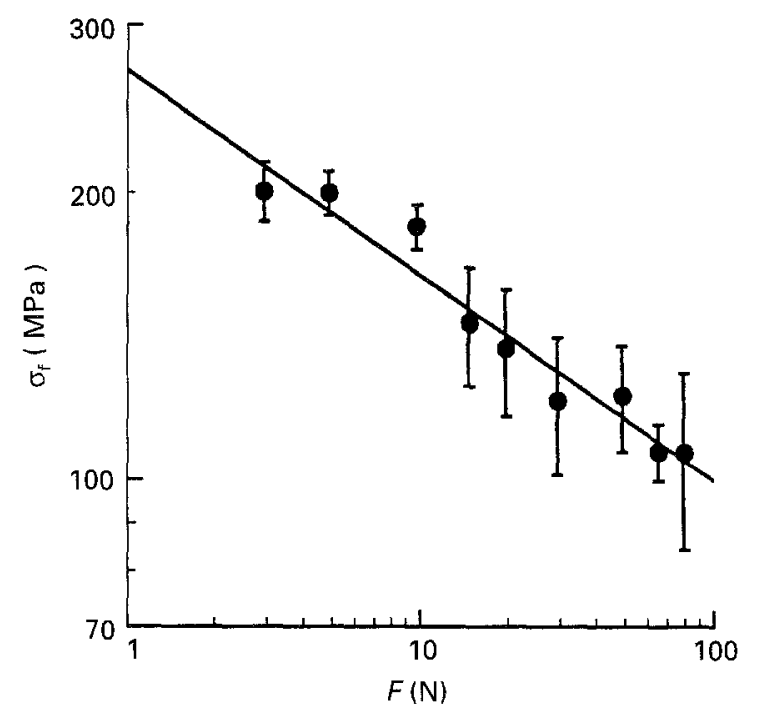

Figure $3 \mathrm{Log}-\log$ plot of fracture stress, $\sigma_{\mathrm{f}}$, versus indentation load, $F$. The least-squares fitted curve has a slope of -0.215 . 
of the minor and major axes also changes. Strictly speaking, $\psi$ varies as these parameters vary, but can be considered constant if the crack size is small compared to the specimen and if the variation of the crack shape during the stable extension is not significant. The value of $\psi$ relevant to the geometry of the MDBT was determined by Chen and Ardell [8] to have the average value 0.97 for our MDBT analysis, using the empirical formula published by Newman and Raju [13]. Although $\psi$ is not constant, as noted previously, the error associated with assuming that it is, has been demonstrated to be small [8]. The value of $\delta$ was calculated using Equation 4 to be 0.0188 . Using the average measured hardness and $E=156 \mathrm{GPa}$, the value of $\chi$ was calculated to be 0.0689 .

Many empirical models for the equilibrium crackgrowth resistance during the growth of indentation cracks have been proposed to describe rising $R$-curve behaviour. For reasons discussed thoroughly elsewhere [8], we use the empirical equation proposed by Cook et al. [14] to describe the resistance to crack extension, $K_{\mathrm{R}}(c)$, as a function of $c$, namely

$$
K_{\mathrm{R}}(c)=K_{\infty}-\frac{Q}{c^{3 / 2}}
$$

where $Q$ is a constant. The merit of this equation is that it contains only two unknown parameters and therefore enables an analytical relationship between $\sigma_{\mathrm{f}}$ and $F$ to be obtained. Using the conditions that determine the onset of unstable crack propagation [15]

$$
K_{\mathrm{e}}\left(c^{*}\right) \geqslant K_{\mathrm{R}}\left(c^{*}\right)
$$

and

$$
\left(\frac{\mathrm{d} K_{\mathrm{e}}}{\mathrm{d} c}\right)_{c=c^{*}} \geqslant\left(\frac{\mathrm{d} K_{\mathrm{R}}}{\mathrm{d} c}\right)_{c=c^{*}}
$$

it is easy to show [6] that $\sigma_{\mathrm{f}}$ depends on $F$ according to the equation

$$
\frac{1}{\sigma_{\mathrm{f}}^{3}}=\chi\left(\frac{4}{K_{\infty}}\right)^{4}\left(\frac{\psi}{3}\right)^{3}\left(F+F^{*}\right)
$$

whereby it becomes apparent that a plot of $1 / \sigma_{\mathrm{f}}^{3}$ versus $F$ should be linear, and that $K_{\infty}$ can be readily obtained from the slope ( $F^{*}$ is a constant).

The fracture toughness was obtained by replotting the data in Fig. 3 in the form $1 / \sigma_{\mathrm{f}}^{3}$ versus $F$ and using Equation 7. The average values of $1 / \sigma_{\mathrm{f}}^{3}$ are plotted versus $F$ in Fig. 4 . There is considerable scatter, but the linearity is good. The fracture toughness was actually evaluated using linear regression analysis of the individual data on the indented specimens, rather than from the data in Fig. 4. The resulting value of $K_{\infty}$ was determined to be $2.69 \pm 0.21 \mathrm{MPa} \mathrm{m}^{1 / 2}$, where the estimated error includes the contributions from the standard deviations of $H$ and $\psi( \pm 0.05)$. Our $\cdot K_{\infty}$ is $28 \%$ larger than the value of $K_{\mathrm{Ic}}=2.1 \mathrm{MPa} \mathrm{m}^{1 / 2}$ reported by Rosenkranz et al. [9]. Fracture was predominantly intergranular, as shown in Fig. 5.

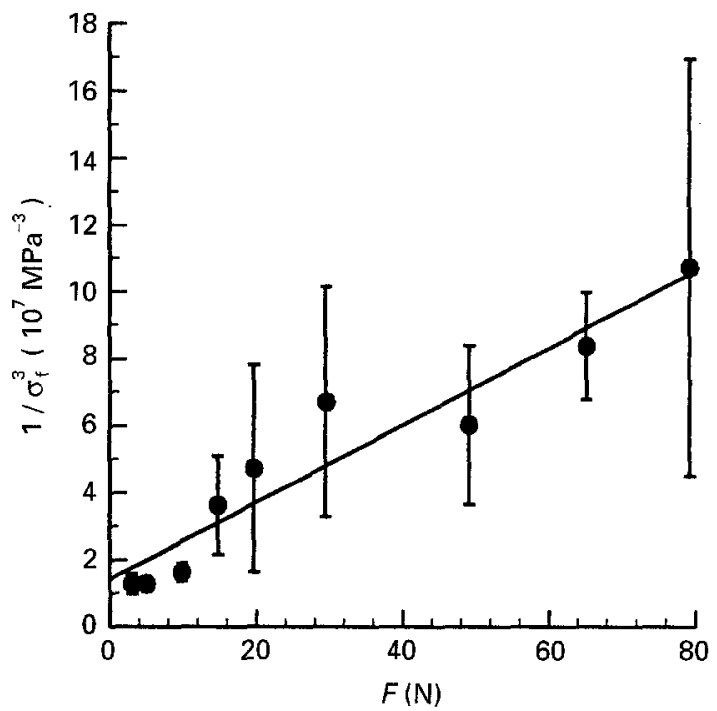

Figure 4 The average values and standard deviations of $1 / \sigma_{\mathrm{f}}^{3}$ plotted against $F$.

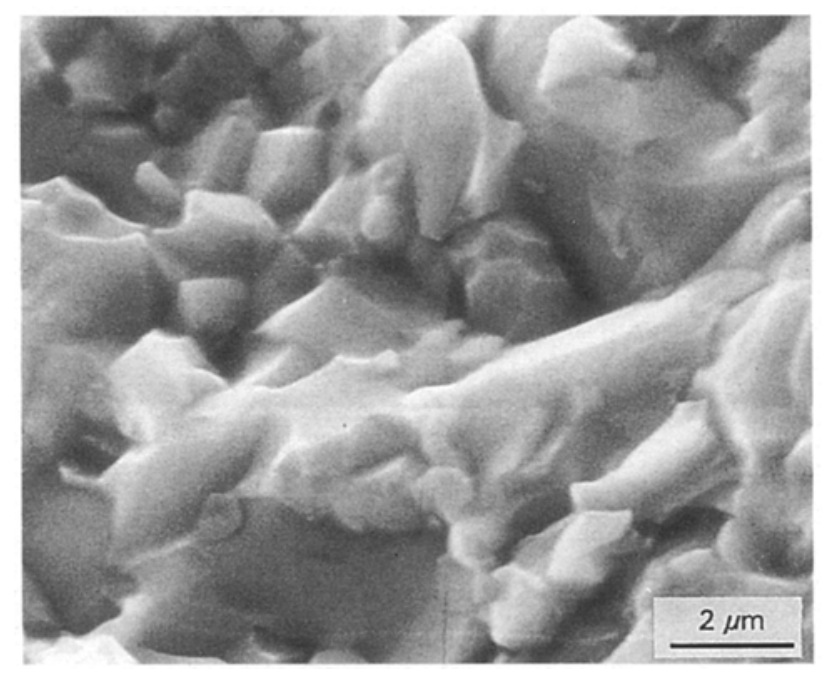

Figure 5 Scanning electron micrograph of the fracture surface of a $\mathrm{Ti}_{5} \mathrm{Si}_{3}$ specimen, illustrating the predominantly intergranular fracture mode.

\section{Discussion}

The empirical $R$-curve described by Equation 5 has been shown to yield satisfactory values of $K_{\infty}$ for ceramics $[6,7,14]$. However, it cannot possibly correctly describe stable crack extension at very small values of $c$ because negative values of $K_{\mathrm{R}}$ are predicted. Another equation that more accurately describes the dependence of $K_{\mathrm{R}}$ on $c$, especially at small values of $c$, has been proposed by Ramachandran and Shetty $[16,17]$. It has the form

$$
K_{\mathrm{R}}=K_{\infty}-\left(K_{\infty}-K_{0}\right) e^{-c / \lambda}
$$

where $K_{0}$ is the fracture resistance at zero crack length and $\lambda$ is a constant. Equation 8 was developed by the fitting of data on apparent fracture toughness as a function of crack length for both $\mathrm{Si}_{3} \mathrm{~N}_{4}$ and $\mathrm{Al}_{2} \mathrm{O}_{3}$ reinforced by $\mathrm{SiC}$ whiskers.

When sufficient data are available on the dependence of fracture toughness on crack length, Equation 8 
appears to provide the most accurate measurements of $K_{\infty}$ [17]. For the type of data generated on numerous ceramics, and of the kind reported herein, where $\sigma_{\mathrm{f}}$ is measured as a function of $F$ and very few measurements of $c^{*}$ have been made, it is important to obtain some sense of whether Equations 5 and 8 yield comparable values of $K_{\infty}$. An analysis of the data of Ramachandran and Shetty [17] was performed by Chen and Ardell [8] using Equation 5, who demonstrated that values of $K_{\infty}$ quite comparable to those published by Ramachandran and Shetty [17] were obtained. Adequate estimates of fracture toughness can therefore be obtained quite simply using the controlledflaw method.

The rising $R$-curve behaviour of $\mathrm{Ti}_{5} \mathrm{Si}_{3}$ is probably due to crack bridging, because other mechanisms are unlikely to be operative. It is difficult to speculate on the reasons for the slightly larger value of the fracture toughness of our material compared to that tested by Rosenkranz et al. [9], because many experimental details were omitted in their brief paper. However, it is known that the fracture toughness of brittle materials is often dependent on grain size, and the difference between the grain sizes of the two different lots of $\mathrm{Ti}_{5} \mathrm{Si}_{3}$ could account for the different values of $K_{\infty}$.

\section{Conclusion}

$\mathrm{Ti}_{5} \mathrm{Si}_{3}$ fractures intergranularly at room temperature and exhibits $R$-curve behaviour. The fracture toughness, as determined using the controlled-flaw method in conjunction with the miniaturized disc-bend test, is $K_{\infty}=2.69 \pm 0.21 \mathrm{MPa} \mathrm{m}^{1 / 2}$. Comparison of this result with previous data on $\mathrm{Ti}_{5} \mathrm{Si}_{3}$ with a larger grain size suggests that refining the grain size might improve the fracture toughness of this material.

\section{Acknowledgements}

The authors thank R. Rosenkranz of the Max Planck Institut Fur Eisenforshchung GMBH for providing the $\mathrm{Ti}_{5} \mathrm{Si}_{3}$ alloy. Partial financial support for this research was provided by the Department of Energy under grant DE-86ER45264.

\section{References}

1. M. SAKAI and R. C. BRADT, Int. Mater. Rev. 38 (1993) 53.

2. P. CHANTIKUL, G. R. ANSTIS, B. R. LAWN and D. B. MARSHALl, $J$. Am. Ceram. Soc. 64 (1981) 539.

3. G. R. ANSTIS, P. CHANTIKUL, B. R. LAWN and D. B. MARSHALL, ibid. 64 (1981) 533.

4. H. LI, F. C. CHEN and A. J. ARDELL, Metall. Trans. 22A (1991) 2061.

5. D. E. MEYERS, F. C. CHEN, J. ZHANG and A. J. ARDELL, J. Test. Eval. 21 (1993) 263.

6. J. ZHANG and A. J. ARDELL, J. Mater. Res. 6 (1991) 1950.

7. Idem, J. Am. Ceram. Soc. 76 (1993) 1340.

8. F. C. CHEN and A. J. ARDELL, Intermetallics, (1994) in press.

9. R. ROSENKRANZ, G. FROMMEYER and W. SMARSLY, Mater. Sci. Eng. A152 (1992) 288.

10. M. N. GIOVAN and G. SINES, J. Am. Ceram. Soc. 62 (1979) 510.

11. B. R. LAWN, A. G. EVANS and D. B. MARSHALL, ibid. 63 (1980) 574.

12. D. K. SHETTY, A. R. ROSENFIELD and W. H. DUCKWORTH., ibid. 68 (1985) C65.

13. J. C. NEWMAN Jr. and I. S. RAJU, Eng. Fract. Mech. 15 (1981) 185

14. R. F. COOK, B. R. LAWN and C. J. FAIRBANKS, J. Am. Ceram. Soc. 68 (1985) 604

15. Y.-W. MAI and B. R. LAWN, Ann. Rev. Mater. Sci. 16 (1986) 415.

16. N. RAMACHANDR.AN and D. K. ShetTY, J. Am. Ceram. Soc. 74 (1991) 2634

17. Idem, J. Mater. Sci. 28 (1993) 6120.

Received 31 October 1994

and accepted 28 April 1995 